\title{
DICRITICAL LOGARITHMIC FOLIATIONS
}

\author{
Felipe Cano and Nuria Corral
}

Abstract

We show the existence of weak logarithmic models for any (dicritical or not) holomorphic foliation $\mathcal{F}$ of $\left(\mathbb{C}^{2}, 0\right)$ without saddlenodes in its desingularization. The models are written in terms of a representative set of separatrices, whose equisingularity types are controlled by the Milnor number of the foliation.

\section{Introduction}

In this paper we show the existence of weak logarithmic models for any holomorphic foliation $\mathcal{F}$ of $\left(\mathbb{C}^{2}, 0\right)$ without saddle-nodes in its desingularization (these foliations are called generalized curves in [2]). If $\mathcal{F}$ is non-dicritical, the existence of a logarithmic model has been proved in $[5]$ and it gives an approximation of $\mathcal{F}$ by a (logarithmic) foliation with linear projective holonomies given by the linear part of the projective holonomies of $\mathcal{F}$.

The first difference in the dicritical case is the choice of the separatrices. Actually, the logarithmic model should be of the form $\eta=0$ for

$$
\eta=\sum_{i=1}^{r} \lambda_{i} \frac{d f_{i}}{f_{i}}
$$

that exhibits the separatrices $f_{i}=0$. But there are several possible ways to do this. For instance the foliation $d\left(\left(y-x^{2}\right) /\left(y+x^{2}\right)\right)=0$ can also be written down as $d\left(y / x^{2}\right)=0$. We choose a representative set $\mathcal{S}$ of separatrices of $\mathcal{F}$ to get explicitly the closed logarithmic form $\eta$. In Theorem 5 it is proved that the possible equisingularity types of $\mathcal{S}$ are bounded by a function of the Milnor number of $\mathcal{F}$. Note also that the

2000 Mathematics Subject Classification. Primary: 32S65.

Key words. Singular foliations, dicritical foliations, logarithmic forms, pencil of curves, singularities.

Partially supported by the Spanish research project MTM 2004-07978 and by the Junta de Castilla y León (VA123/04). 
results in this paper would provide a normalized presentation of a pencil of curves, in terms of multivalued functions.

To have a weak logarithmic model $\mathcal{L}$ for $\mathcal{F}$ roughly speaking means that the reduction of the singularities of $\mathcal{L}$ is longer than the one of $\mathcal{F}$ and coincides with it outside a "escape set" of non-singular points for $\mathcal{F}$ placed at dicritical components (domination of foliations). The escape set can be chosen to be a singleton at such dicritical components and this determines the residues $\lambda_{i}$. We prove (Theorem 14) that this concept depends in fact only on the $\mathbb{C}$-divisor $(\mathcal{S}, \lambda)$ and not on the particular logarithmic foliation we take. This is key in the proof of the existence of weak logarithmic models (Theorems 3 and 16) since it serves to "glue" the models by induction.

We end the paper by a list of examples showing the difficulties in order to get a logarithmic model without escape set.

\section{Domination of foliations. Representative sets of separatrices}

We consider holomorphic singular foliations $\mathcal{F}$ defined on ambient spaces $M$ of dimension two, equipped with a normal crossing divisor $D \subset M$. As usual, we say that a component $F$ of $D$ is dicritical if it is generically transversal to $\mathcal{F}$; if $F$ is invariant, we say that it is non-dicritical.

The reduction of singularities of $\mathcal{F}$ at $P \in M$ can be done relatively to $D$ as follows. Denote $\operatorname{Sing}(\mathcal{F}, D)$ the set of points $Q \in M$ where $\mathcal{F}$ and $D$ do not have normal crossings; that is, either $Q \in \operatorname{Sing} \mathcal{F}$ or $Q \notin \operatorname{Sing} \mathcal{F}$ but the only invariant curve of $\mathcal{F}$ at $Q$ does not have normal crossings with $D$. In the case $D=\emptyset$, note that $\operatorname{Sing}(\mathcal{F}, \emptyset)=\operatorname{Sing} \mathcal{F}$. We say that $P \in \operatorname{Sing}(\mathcal{F}, D)$ is simple for $(\mathcal{F}, D)$ iff $P \in D$, it is a simple singularity in the sense of Seidenberg [10], [12] and each component of $D$ through $P$ is non-dicritical. Then we have

Theorem (Reduction of singularities $[\mathbf{3}],[\mathbf{1 2}])$. Let $(M, P)$ be the germ of $M$ at $P$. There is a finite composition of blowing-ups $\pi=$ $\pi_{(\mathcal{F}, D, P)}: M^{\prime} \longrightarrow(M, P)$ such that, if $\mathcal{F}^{\prime}$ is the transform of $\mathcal{F}$ by $\pi$ and $D^{\prime}=\pi^{-1}(D \cup\{P\})$, then any $Q \in \operatorname{Sing}\left(\mathcal{F}^{\prime}, D^{\prime}\right)$ is simple and $\pi$ is minimal in the sense that it cannot be factorized by another morphism with the above property.

We call $\pi_{(\mathcal{F}, D, P)}$ the minimal reduction of singularities of $(\mathcal{F}, D)$ at $P$. To get it it's enough to blow-up successively the non-simple points in $\operatorname{Sing}(\mathcal{F}, D)$ (the processus stops by similar arguments to the ones 
in $[\mathbf{1 2}])$. Note that if $D=\emptyset$ the reduction $\pi_{(\mathcal{F}, \emptyset, P)}$ is maybe longer than Seidenberg's reduction; the main difference appears because of the tangencies with the created dicritical components. If $\mathcal{F}$ is non-dicritical (each succesive blowing-up is non-dicritical) and $D=\emptyset$, we get essentially the same reduction of singularities as Seidenberg. Even if $\mathcal{F}$ is non-dicritical, the dicritical components of the starting divisor $D$ may produce a longer reduction of singularities; unfortunately, we have to keep them in this paper in order to assure coherence in our induction statements.

Notation. Since $\mathcal{F}, D$ and its reduction of singularities will be fixed, in all this paper we keep the notations $\pi=\pi_{(\mathcal{F}, D, P)}, E^{\prime}=\pi^{-1}(P), D^{\prime}=$ $\pi^{-1}(D \cup\{P\})$ and we denote $\mathcal{F}^{\prime}, \mathcal{L}^{\prime}, \mathcal{G}^{\prime}$ the transforms of the foliations $\mathcal{F}, \mathcal{L}, \mathcal{G}$ by $\pi$. For a germ of analytic curve $\Gamma$ at $P$, we will denote by $\Gamma^{\prime}$ the strict transform of $\Gamma$ by $\pi$. Also we shall keep the notation $\pi_{1}: M_{1} \rightarrow(M, P)$ for the blowing-up of $(M, P)$ with center $P$ and we will denote $E_{1}=\pi_{1}^{-1}(P)$ the exceptional divisor, $D_{1}=\pi_{1}^{-1}(D \cup\{P\})$ and $\mathcal{F}_{1}, \mathcal{L}_{1}, \mathcal{G}_{1}, \Gamma_{1}$ the transforms of objects as above.

Let $\mathcal{G}$ be another singular foliation on $M$ and assume that $P$ is a simple singularity for both $(\mathcal{F}, D)$ and $(\mathcal{G}, D)$. We say that $P$ has same linear type of singularity for $(\mathcal{F}, D)$ and $(\mathcal{G}, D)$ if $I_{P}(\mathcal{F}, F)=I_{P}(\mathcal{G}, F)$ for any component $F$ of $D$ through $P$, where $I_{P}(\mathcal{F}, F)$ is the Camacho-Sad index (see $[\mathbf{1}]$ ). In this paper we exclude saddle-nodes at the end of the desingularization, that is, we restrict ourselves to generalized curves as defined in [2]; in this case, the indices at simple singularities correspond to the two quotients of eigenvalues.

Definition 1. We say that $(\mathcal{G}, D)$ dominates $(\mathcal{F}, D)$ at $P$ if:

(1) The components of $D^{\prime}$ are simultaneously dicritical or non-dicritical for the transforms $\mathcal{F}^{\prime}$ and $\mathcal{G}^{\prime}$ of $\mathcal{F}, \mathcal{G}$ by $\pi$.

(2) For any non-dicritical component $F^{\prime}$ of $D^{\prime}$ we have that

$$
\operatorname{Sing}\left(\mathcal{F}^{\prime}, D^{\prime}\right) \cap F^{\prime}=\operatorname{Sing}\left(\mathcal{G}^{\prime}, D^{\prime}\right) \cap F^{\prime}
$$

and each $Q \in \operatorname{Sing}\left(\mathcal{F}^{\prime}, D^{\prime}\right)$ is a simple singularity for $\left(\mathcal{G}^{\prime}, D^{\prime}\right)$ with same linear type and same separatrices as for $\left(\mathcal{F}^{\prime}, D^{\prime}\right)$.

Note that in the case $D=\emptyset$ and $\mathcal{F}$ non-dicritical, we get that $\pi_{(\mathcal{F}, \emptyset, P)}=\pi_{(\mathcal{G}, \emptyset, P)}$ and $\mathcal{F}, \mathcal{G}$ have the same set of separatrices.

Definition 2. Let $\Gamma$ be a separatrix for $\mathcal{F}$ at $P$ not contained in $D$. Following $[\mathbf{2}]$, we say that $\Gamma$ is isolated for $(\mathcal{F}, D)$ if the strict transform $\Gamma^{\prime}$ does not cut $E^{\prime}$ in a $\mathcal{F}^{\prime}$-dicritical component. We say that $\Gamma$ is an 
$F^{\prime}$-curvette for $\mathcal{F}$ if $\Gamma^{\prime}$ cuts $E^{\prime}$ in a $\mathcal{F}^{\prime}$-dicritical component $F^{\prime}$. A set $\mathcal{S}$ of separatrices of $\mathcal{F}$ at $P$ is representative for $(\mathcal{F}, D)$ if it contains the isolated separatrices, the non-dicritical components of $D$ at $P$ and the number of $F^{\prime}$-curvettes in $\mathcal{S}$ for each dicritical component $F^{\prime}$ of $E^{\prime}$ is equal to $\max \left\{0,2-v_{D^{\prime}} F^{\prime}\right\}$, where the valence $v_{D^{\prime}} F^{\prime}$ is the number of components of $D^{\prime}$ that intersect $F^{\prime}$ (excluding $F^{\prime}$ ).

Note that if $\mathcal{F}$ is non-dicritical the only representative set of separatrices for $(\mathcal{F}, D)$ is the set of all separatrices. In the case of a dicritical $\mathcal{F}$ we know that we have infinitely many separatrices. In order to get a logarithmic model we have to choose finitely many of them. The representative sets of separatrices give our choice. The idea is to take not too much "curvettes" to assure that the equisingularity types of the chosen set of separatrices remain bounded by the Milnor number of the foliation (Theorem 5).

This paper is devoted to prove the following theorem:

Theorem 3. Let $\mathcal{F}$ be a generalized curve of $\left(\mathbb{C}^{2}, 0\right)$ and $\mathcal{S}=\left\{\Gamma^{i}\right\}_{i=1}^{r}$ a representative set of separatrices for $\mathcal{F}$. Take a local equation $f_{i}=0$ of $\Gamma^{i}$ for $i=1,2, \ldots, r$. Then there are $\lambda_{i} \in \mathbb{C}^{*}, i=1,2, \ldots, r$, such that if

$$
\eta=\sum_{i=1}^{r} \lambda_{i} \frac{d f_{i}}{f_{i}}
$$

the logarithmic foliation $\eta=0$ dominates $\mathcal{F}$.

Let us explain with two examples the definition of a representative set of separatrices. Consider the foliation (Suzuki's example, see [4]) $\mathcal{F}$ defined by $\omega=\left(2 y^{2}+x^{3}\right) d x-2 x y d y=0$. It has only one isolated separatrix given by $y^{2}-x^{3}=0$. Thus it is not possible to construct a logarithmic foliation written only in terms of the isolated separatrices and dominating $\mathcal{F}$ since it will be non-dicritical. To obtain a logarithmic foliation that dominates $\mathcal{F}$ it is enough to consider the foliation $d\left(\left(y^{2}-x^{3}\right) / x^{2}\right)=0$. Note that $S=\left\{y^{2}-x^{3}=0, x=0\right\}$ is a representative set of separatrices of $\mathcal{F}$. The second example is given by the foliations $\mathcal{F}_{p, q}$ defined by $p x d y-q y d x=0$ with $p, q \in \mathbb{N}$. If $p, q \geq 2$ and $\operatorname{gcd}(p, q)=1$, then the only dicritical component in the reduction of singularities of $\mathcal{F}_{p, q}$ has valence 2 . The curvettes of this dicritical component are the curves $y^{p}-c x^{q}=0$ with $c \in \mathbb{C}$. However, the foliation $\mathcal{F}_{p, q}$ is given by $d\left(y^{p} / x^{q}\right)=0$ where $\mathcal{S}=\{x=0, y=0\}$ are the isolated separatrices and $\mathcal{S}$ is a representative set of separatrices. Note also that the type of equisingularity of the curves $y^{p}-c x^{q}=0$ is not bounded by a function of the Milnor number since $\mu\left(\mathcal{F}_{p, q}\right)=1$.

Let us give now some properties of a representative set of separatrices. 
Proposition 4. Let $\mathcal{F}$ be a generalized curve at $P \in M, D$ a normal crossings divisor and $\mathcal{S}=\left\{\Gamma^{i}\right\}_{i=1}^{r}$ a representative set of separatrices for $(\mathcal{F}, D)$. Denote by $C_{P} \mathcal{S} \subset E_{1}$ the tangent cone of $\mathcal{S}$. Then we have

(1) $\mathcal{S} \neq \emptyset$, that is $r \geq 1$.

(2) If $\mathcal{S}$ has only one element and it is non-singular at $P$, then $P \notin$ Sing $\mathcal{F}$.

(3) If $\pi_{1}$ is non-dicritical for $\mathcal{F}$, then $C_{P} \mathcal{S}=\operatorname{Sing} \mathcal{F}_{1}=\operatorname{Sing}\left(\mathcal{F}_{1}, D_{1}\right)$.

(4) If $\pi_{1}$ is $\mathcal{F}$-dicritical, then $\operatorname{Sing}\left(\mathcal{F}_{1}, D_{1}\right) \subset C_{P} \mathcal{S}$.

(5) If $\pi_{1}$ is $\mathcal{F}$-dicritical, then $C_{P} \mathcal{S}$ has $\max \left\{2, v_{D^{\prime}} E_{1}\right\}$ points.

Proof: (1) If $\mathcal{F}$ is non-dicritical, the result follows from [1]. Note also that the arguments in [1] imply that any connected component of the union of non-dicritical components of the exceptional divisor after reduction of singularities will support at least one isolated separatrix of $\mathcal{F}$, which is necessarily an element of $\mathcal{S}$. Thus, it remains to consider the case that all the components of the exceptional divisor are dicritical; since two such components do not intersect, there is only one of them, we are in the radial case and $\mathcal{S}$ has two elements.

(2) If $\mathcal{F}$ is non-dicritical, the result follows from [2], since $\mathcal{F}$ has the same reduction of singularities as the set of separatrices. Moreover, if $\mathcal{S}$ has only one element, then $\mathcal{F}$ must be non-dicritical. To see this, note first that $\mathcal{F}$ is not radial, and hence, by the above arguments, the only element of $\mathcal{S}$ is an isolated separatrix of $\mathcal{F}$, thus there are no "curvettes" in $\mathcal{S}$. Then, there is a dicritical component $F^{\prime}$ with valence greater or equal than two. Now $F^{\prime}$ cuts at least two connected components of the union of non-dicritical components. This implies the existence of at least two elements in $\mathcal{S}$ in view of the arguments in (1).

(3) It is evident that $C_{P} \mathcal{S} \subset \operatorname{Sing} \mathcal{F}_{1}$, since $\mathcal{F}_{1}$ has at least two separatrices at the points in $C_{P} \mathcal{S}$ : the exceptional divisor $E_{1}$ and the strict transform of an element of $\mathcal{S}$. To see that $\operatorname{Sing} \mathcal{F}_{1}=\operatorname{Sing}\left(\mathcal{F}_{1}, D_{1}\right)$, take a point $Q \notin \operatorname{Sing} \mathcal{F}_{1}$, then either $D_{1}=E_{1}$ locally at $Q$ or $D_{1}=E_{1} \cup F$ and in both cases we have the normal crossings property, since $E_{1}$ is invariant. It remains to show that if $Q \notin C_{P} \mathcal{S}$, then $Q \notin \operatorname{Sing} \mathcal{F}_{1}$, but this follows from (2) since the only separatrix of $\mathcal{F}_{1}$ at $Q$ is the exceptional divisor $E_{1}$.

(4) Take $Q \in \operatorname{Sing}\left(\mathcal{F}_{1}, D_{1}\right)$. Since $E_{1}$ is dicritical, then $Q$ is not a simple singularity for $\left(\mathcal{F}_{1}, D_{1}\right)$ and thus the reduction of singularities continues through $Q$. By (1), we get an element of $\mathcal{S}$ passing through $Q$.

(5) We have that

$$
C_{P} \mathcal{S}=C_{P}\left\{E_{1} \text {-curvettes in } \mathcal{S}\right\} \cup C_{P} D \cup \operatorname{Sing}\left(\mathcal{F}_{1}, D_{1}\right) \text {. }
$$


Moreover, since the points in $\operatorname{Sing}\left(\mathcal{F}_{1}, D_{1}\right)$ are not simple singularities, we get that the valence $v_{D^{\prime}} E_{1}$ is equal to the number of points in $C_{P} D \cup$ $\operatorname{Sing}\left(\mathcal{F}_{1}, D_{1}\right)$. The result follows noting that if $v_{D^{\prime}} E_{1} \geq 2$ there are no $E_{1}$-curvettes in $\mathcal{S}$.

Theorem 5. There is a finite set $\mathcal{E}(\mu)$ for each integer number $\mu \geq 0$ such that the following property holds. Denote by $\mu_{P} \mathcal{F}$ the Milnor number of $\mathcal{F}$. Then the equisingularity type of $\mathcal{S}$ is in $\mathcal{E}\left(\mu_{P} \mathcal{F}\right)$, for any generalized curve $\mathcal{F}$ and a normal crossings divisor $D$ at $P \in M$, where $\mathcal{S}=\left\{\Gamma^{i}\right\}_{i=1}^{r}$ is any representative set of separatrices for $(\mathcal{F}, D)$.

Proof: By a theorem in [6], the equisingularity types of the union $S^{*}$ of isolated separatrices and non-dicritical components of $D$ are bounded (in the sense of the statement) by a function of the Milnor number. Now, to get the equisingularity type of a representative set of separatrices we have just to add at most one (or two arrows in the radial case) arrow to the terminal vertices of the dual graphs representing each equisingularity type for $S^{*}$.

\section{Closed logarithmic forms and $\mathbb{C}$-divisors}

Consider a non-empty finite set $\mathcal{S}=\left\{\Gamma^{i}\right\}_{i=1}^{r}$ of germs of irreducible curves at $(M, P)$ and take a list $\lambda=\left\{\lambda_{i}\right\}_{i=1}^{r}$ with $\lambda_{i} \in \mathbb{C}^{*}$. The pair $\mathcal{D}=(\mathcal{S}, \lambda)$ is called a $\mathbb{C}$-divisor at $P$ and its support is $\operatorname{Supp} \mathcal{D}=\cup_{i=1}^{r} \Gamma^{i}$. Write $m_{i}=\nu_{P} \Gamma^{i}$ the multiplicity of $\Gamma^{i}$ at $P$. We say that $P$ is nonsingular for $\mathcal{D}$ if $r=1$ and $m_{1}=1$. We say that $P$ is a simple singularity for $\mathcal{D}$ if $r=2$ and $m_{1}=m_{2}=1$, the curves $\Gamma^{1}$ and $\Gamma^{2}$ have distinct tangents and $\lambda_{1} / \lambda_{2} \notin \mathbb{Q}<0$. More generally, these definitions can be made relative to a normal crossings divisor $D \subset M$ as follows. We say that $P$ is non-singular for $(\mathcal{D}, D)$ if it is non-singular for $\mathcal{D}$ and $\Gamma^{1}$ has normal crossings with $D$ and we say that it is a simple singularity for $(\mathcal{D}, D)$ if it is a simple singularity for $\mathcal{D}$ and $\emptyset \neq D \subset \Gamma^{1} \cup \Gamma^{2}$.

Let us define the transform $\mathcal{D}_{Q}=\left(\mathcal{S}_{Q}, \lambda_{Q}\right)$ of $\mathcal{D}$ at a point $Q \in E_{1}$ under the blowing-up $\pi_{1}: M_{1} \rightarrow(M, P)$. Denote $I_{Q}$ the set of indices defined by the property that $Q \in \Gamma_{1}^{i}$ for $i \in I_{Q}$ and put $\lambda_{0}=\sum_{i=1}^{r} m_{i} \lambda_{i}$. Then

$$
\begin{array}{ll}
\mathcal{S}_{Q}=\left\{\Gamma_{1}^{i}\right\}_{i \in I_{Q}}, \lambda_{Q}=\left\{\lambda_{i}\right\}_{i \in I_{Q}}, & \text { if } \lambda_{0}=0 . \\
\mathcal{S}_{Q}=\left\{\Gamma_{1}^{0}=E_{1}\right\} \cup\left\{\Gamma_{1}^{i}\right\}_{i \in I_{Q}}, \lambda_{Q}=\left\{\lambda_{0}\right\} \cup\left\{\lambda_{i}\right\}_{i \in I_{Q}}, & \text { if } \lambda_{0} \neq 0 .
\end{array}
$$

Note that if $\mathcal{S}_{Q}=\emptyset$ then $Q \notin \operatorname{Supp} \mathcal{D}_{Q}$. This construction can be iterated by blowing-up at points in the support. To be precise, we say that a finite composition of blowing-ups $\sigma: M^{*} \rightarrow(M, P)$ is a $\mathcal{D}$-admissible 
morphism if the centers of the blowing-ups are in the support of the corresponding transformed $\mathbb{C}$-divisors. Moreover, we have a reduction of singularities as follows

Proposition 6. Let $\mathcal{D}=(\mathcal{S}, \lambda)$ be a $\mathbb{C}$-divisor and $D$ a normal crossings divisor on $(M, P)$. There is a $\mathcal{D}$-admissible morphism $\tilde{\pi}=\pi_{(\mathcal{D}, D, P)}: \tilde{M} \rightarrow$ $(M, P)$ such that any point $Q \in \operatorname{Supp} \mathcal{D}_{\tilde{\pi}}$ in the support of the transformed $\mathbb{C}$-divisor is non-singular or simple for $\left(\mathcal{D}_{\tilde{\pi}}, \tilde{D}\right)$, where $\tilde{D}=$ $\tilde{\pi}^{-1}(\{P\} \cup D)$ and $\tilde{\pi}$ is minimal in the sense that it cannot be factorized by another morphism with the above property.

The proof is a consequence of the reduction of singularities of curves and Euclides' algorithm, very similar (but simpler) to the proof in [12].

Consider reduced equations $f_{i}=0$ of $\Gamma^{i}$ for $i=1,2, \ldots, r$ and put $f=f_{1} f_{2} \cdots f_{r}$. We say that a closed meromorphic 1 -form $\eta$ is $(\mathcal{S}, \lambda)$ logarithmic if

$$
\eta=\sum_{i=1}^{r} \lambda_{i} \frac{d f_{i}}{f_{i}}+\alpha,
$$

where $\alpha$ is holomorphic. In particular $\alpha$ is closed and hence $\alpha=d h$. In fact, we can write $\eta=\sum_{i=1}^{r} \lambda_{i} d f_{i}^{*} / f_{i}^{*}$ for suitable local equations $f_{i}^{*}=0$ of each $\Gamma^{i}$ (to see this, put $\alpha=d u / u$, where $u(P) \neq 0$ ). Note that if $\eta$ is $(\mathcal{S}, \lambda)$-logarithmic, then $\pi_{1}^{*} \eta$ is $\left(\mathcal{S}_{Q}, \lambda_{Q}\right)$-logarithmic at $Q \in E_{1}$.

Definition 7. We say that $\mathcal{D}=(\mathcal{S}, \lambda)$ is a 1 -faithful $\mathbb{C}$-divisor at $P$ if there is a $\mathcal{D}$-logarithmic 1 -form $\eta$ such that

$$
\nu_{P}(f \eta)=m-1,
$$

where $m=\nu_{P} f=\sum_{i=1}^{r} m_{i}$. (A direct computation shows that this will be then true for any $\mathcal{D}$-logarithmic 1 -form). We say that $\mathcal{D}$ is faithful if its transform is 1-faithful at any point of the support after any $\mathcal{D}$-admissible morphism.

The non-singular points and the simple singularities are persistent under blowing-up and also the $\mathbb{C}$-divisor is faithful at this kind of points. Thus in order to verify the faithfulness of $\mathcal{D}$ is enough to verify the 1 -faithfulness at the intermediary points in the reduction of singularities.

Lemma 8. Consider the $\mathbb{C}$-divisor $\mathcal{D}=(\mathcal{S}, \lambda)$ and let $\eta$ be a $\mathcal{D}$-logarithmic 1-form. Denote by $\mathcal{L}^{\eta}$ the foliation $\eta=0$. Put $\lambda_{0}=\sum_{i=1}^{r} m_{i} \lambda_{i}$, as above.

a) If $\lambda_{0} \neq 0$, then $\pi_{1}$ is non-dicritical for $\mathcal{L}^{\eta}$ and $\mathcal{D}$ is 1-faithful.

b) If $\lambda_{0}=0$ and $\mathcal{D}$ is 1-faithful, then $\pi_{1}$ is dicritical for $\mathcal{L}^{\eta}$. 
Proof: First, let us recall that if a foliation $\mathcal{G}$ is given at $P$ by $\omega=0$, where $\omega=a(x, y) d x+b(x, y) d y$ is a germ of holomorphic form of order $t$, then $\pi_{1}$ is dicritical for $\mathcal{G}$ if and only if $x \operatorname{In}^{t} a+y \operatorname{In}^{t} b=0$, where $\operatorname{In}^{t} a$, $\operatorname{In}^{t} b$ denote the parts of degree $t$ of $a, b$ (here $a$ and $b$ are not necessarily without common factor). Now the part a) of the lemma is an immediate computation, since $f \eta$ is a holomorphic 1 -form that gives $\mathcal{L}^{\eta}$. To prove part b), it is enough to note that the fact that $\mathcal{D}$ is 1 -faithful means that the initial part of $f \eta$ has degree $m-1$ and then the condition $\lambda_{0}=0$ is equivalent to the dicriticalness of $\pi_{1}$.

As a consequence, if $\lambda_{0}=0$ and $\pi_{1}$ is non-dicritical, the divisor $\mathcal{D}$ fails to be 1-faithful.

Example 9. Taking $\mathcal{S}=\left\{y-x^{2}=0, y+x^{2}=0\right\}$ and $\lambda=(1,-1)$ we get a non 1-faithful $\mathcal{D}=(\mathcal{S}, \lambda)$. In fact $\lambda_{0}=0$ and $\pi_{1}$ is non-dicritical for the foliation $d\left(\left(y-x^{2}\right) /\left(y+x^{2}\right)\right)=0$. Note that the same foliation is given by $d\left(y / x^{2}\right)=0$ and this presentation is faithful. More generally, if the curves of $\mathcal{S}$ have all the same tangent and $\lambda_{0}=0$, we have a non-dicritical blowing-up, as we show next.

Proposition 10. Assume that the curves of $\mathcal{S}=\left\{\Gamma^{i}\right\}_{i=1}^{r}$ have all the same tangent at $P$ and that $\lambda_{0}=0$. Take any $(\mathcal{S}, \lambda)$-logarithmic form $\eta$. Then $\pi_{1}$ is non-dicritical for the logarithmic foliation $\mathcal{L}$ given by $\eta=0$.

Proof: Put $f_{i}(x, y)=y^{m_{i}}+h_{i}(x, y)$ with $\nu_{P}\left(h_{i}\right)>m_{i}$ for $i=1,2, \ldots, r$. Take coordinates $(u, v)$ in the second chart of the blowing-up such that $\pi_{1}(u, v)=(u v, v)$. We can write $f_{i}=f_{i}(u v, v)=v^{m_{i}}\left(1+F_{i}(u, v)\right)$ with $F_{i}(u, 0)=0$ and then we have

$$
\begin{aligned}
\frac{d f_{i}}{f_{i}} & =m_{i} \frac{d v}{v}+\frac{d F_{i}}{1+F_{i}}, \\
\pi_{1}^{*} \eta & =\sum_{i=1}^{r} \lambda_{i} \frac{d F_{i}}{1+F_{i}}=A(u, v) \frac{d u}{u}+B(u, v) \frac{d v}{v} .
\end{aligned}
$$

Let us put $M=\max \left\{k: v^{k}\right.$ divides $A$ and $\left.B\right\}, A(u, v)=v^{M} \tilde{A}(u, v)$ and $B(u, v)=v^{M} \tilde{B}(u, v)$. To show that $v=0$ is invariant by $\pi_{1}^{*} \eta=0$ is enough to verify that $\tilde{B}(u, 0) \neq 0$. Since $\pi_{1}^{*} \eta$ is a closed form, we have

$$
v \frac{\partial A}{\partial v}=u \frac{\partial B}{\partial u} .
$$

If $v^{k}$ divides $B$, it must also divide $u \partial B / \partial u$ and consequently $v^{k}$ divides $v \partial A / \partial v$. The hypothesis over the $F_{i}$ imply that $A(u, 0)=0$ and thus $v^{k}$ divides $A$. We conclude that $M=\max \left\{k: v^{k}\right.$ divides $\left.B\right\}$ and then $\tilde{B}(u, 0) \neq 0$. 
Remark 11 (Mattei). This proof corresponds to the fact that a multivalued or meromorphic function in $\mathbb{P}_{\mathbb{C}}^{1}$ with at most either one zero or one pole is constant.

Corollary 12. Let $\mathcal{D}=(\mathcal{S}, \lambda)$ be 1-faithful. Then $\operatorname{Supp} \mathcal{D}_{\pi_{1}}$ has at least two points in the exceptional divisor $E_{1}$ of the first blowing-up $\pi_{1}$. As a consequence, if $\mathcal{D}$ is faithful and $\sigma: M^{*} \rightarrow(M, P)$ is any $\mathcal{D}$-admissible morphism, then each component $F^{*}$ of $E^{*}=\sigma^{-1}(P)$ has at least two points in $\operatorname{Supp} \mathcal{D}_{\sigma}$.

In the next proposition we recover the relationship between nondicritical components and support components.

Proposition 13. Let $\mathcal{D}=(\mathcal{S}, \lambda)$ be a faithful $\mathbb{C}$-divisor, consider a $\mathcal{D}$ logarithmic form $\eta$, and the corresponding foliation $\mathcal{L}^{\eta}$. Let $\sigma: M^{*} \rightarrow$ $(M, P)$ be any $\mathcal{D}$-admissible morphism. Then a component $F^{*}$ of $E^{*}=$ $\sigma^{-1}(P)$ is non-dicritical for the transform $\mathcal{L}_{\sigma}^{\eta}$ if and only if $F^{*}$ is contained in the support of the transformed $\mathbb{C}$-divisor $\mathcal{D}_{\sigma}$.

Proof: It follows just applying the properties of 1-faithfulness at each center in the sequence of blowing-ups.

\section{Existence of weak logarithmic models}

In this section, we consider a generalized curve $\mathcal{F}$ at $P$, a normal crossings divisor $D \subset M$ and a representative set of separatrices $\mathcal{S}=$ $\left\{\Gamma^{i}\right\}_{i=1}^{r}$ for $(\mathcal{F}, D)$ at $P$. We say that a $\mathbb{C}$-divisor $\mathcal{D}=(\mathcal{S}, \lambda)$ is a weak logarithmic model for $(\mathcal{F}, D)$ at $P$, relatively to $\mathcal{S}$ if and only if we have the following properties:

(1) $\mathcal{D}$ is faithful.

(2) $\pi_{(\mathcal{D}, D, P)}=\pi_{(\mathcal{F}, D, P)}$.

(3) Put $\pi=\pi_{(\mathcal{F}, D, P)}: M^{\prime} \rightarrow(M, P)$. Then any component $F^{\prime}$ of $E^{\prime}$ is dicritical for $\mathcal{F}^{\prime}$ if and only if $F^{\prime}$ is not contained in the support $\operatorname{Supp} \mathcal{D}_{\pi}$.

(4) Any point $Q \in \operatorname{Sing}\left(\mathcal{F}^{\prime}, D^{\prime}\right)$ is in the support $\operatorname{Supp} \mathcal{D}_{\pi}$ and the quotient of residues $-\lambda_{Q}^{2} / \lambda_{Q}^{1}$ is the quotient of the eigenvalues of $\mathcal{F}^{\prime}$ at $Q$.

The fact that $\mathcal{D}=(\mathcal{S}, \lambda)$ is a weak logarithmic model for $(\mathcal{F}, D)$ depends only on the projective class $[\lambda] \in \mathbb{P}_{\mathbb{C}}^{r-1}$. Note that $P \in \operatorname{Supp} \mathcal{D}$. Moreover, the definition is compatible with the blowing-ups in the following sense. Take any point $Q \in E_{1}$ in the support $\operatorname{Supp} \mathcal{D}_{\pi_{1}}$, then $\mathcal{D}_{\pi_{1}}$ is a weak logarithmic model for $\left(\mathcal{F}_{1}, D_{1}\right)$ at $Q$. 
Theorem 14. Assume that $\mathcal{D}=(\mathcal{S}, \lambda)$ is a weak logarithmic model for $(\mathcal{F}, D)$ at $P$, take any $\mathcal{D}$-logarithmic form $\eta$ and let $\mathcal{L}^{\eta}$ be the foliation $\eta=0$. Then $\left(\mathcal{L}^{\eta}, D\right)$ dominates $(\mathcal{F}, D)$ at $P$.

Proof: Let us do induction on the number $N_{P}(\mathcal{F}, D)$ of blowing-ups needed to desingularize $(\mathcal{F}, D)$ at $P$. Let us consider the case $N_{P}(\mathcal{F}, D)=$ 0 . Assume first that $P \notin \operatorname{Sing} \mathcal{F}$. Then the set $\mathcal{S}$ has only one element $\Gamma$ that is non-singular at $P$. Take coordinates $x, y$ such that $\Gamma=\{x=0\}$ and $D \subset\{x y=0\}$. Note that $\mathcal{D}=\left(\mathcal{S},\left\{\lambda_{1}\right\}\right)$ and $x \eta=d x+x \alpha$, for a closed holomorphic form $\alpha$. Then obviously $\left(\mathcal{L}^{\eta}, D\right)$ dominates $(\mathcal{F}, D)$ at $P$. Assume now that $P \in \operatorname{Sing} \mathcal{F}$. Then up to a choice of coordinates we have that $\mathcal{S}=\left\{\Gamma_{1}, \Gamma_{2}\right\}$, with $\Gamma_{1}=\{x=0\}, \Gamma_{2}=\{y=0\}$ and $\Gamma_{1} \subset D \subset \Gamma_{1} \cup \Gamma_{2}$. Moreover $-\lambda_{2} / \lambda_{1} \notin \mathbb{Q}_{>0}$ is the quotient of eigenvalues for $\mathcal{F}$. Then $x y \eta=\lambda_{1} y d x+\lambda_{2} x d y+x y \alpha$, for a closed holomorphic form $\alpha$. Then $P$ is a simple singularity for $\left(\mathcal{L}^{\eta}, D\right)$ with separatrices $x y=0$ and same quotient of eigenvalues as $\mathcal{F}$; the domination is straightforward.

Let us do the induction step. Assume first that $\pi_{1}$ is non-dicritical for $\mathcal{F}$. Hence $\lambda_{0} \neq 0$ and $\pi_{1}$ is non-dicritical for $\mathcal{L}^{\eta}$. Note also that $E_{1} \subset \operatorname{Supp} \mathcal{D}_{\pi_{1}}$. Take a component $F$ of $D$, to verify if $F$ is dicritical or not for $\mathcal{L}^{\eta}$ is enough to do it for the strict transform $F_{1}$ of $F$ with respect to $\mathcal{L}_{1}^{\eta}$; this one and the rest of the conditions for the domination property can be verified at the points $Q \in E_{1}$ and this is given by the induction hypothesis.

Assume now that $\pi_{1}$ is dicritical for $\mathcal{F}$. Then $\lambda_{0}=0$ and in view of the faithfulness $\pi_{1}$ is dicritical for $\mathcal{L}^{\eta}$. Moreover $\operatorname{Supp} \mathcal{D}_{\pi_{1}} \cap E_{1}=C_{P} \mathcal{S}$. Take a component $F$ of $D$, if $F$ is non-dicritical for $\mathcal{F}$, then $F \in \mathcal{S}$ and hence $F$ is non-dicritical for $\mathcal{L}^{\eta}$. Assume that $F$ is dicritical for $\mathcal{F}$ and put $Q_{1}=F_{1} \cap E_{1}$, then $Q_{1} \in \operatorname{Sing}\left(\mathcal{F}_{1}, D_{1}\right) \subset C_{P} \mathcal{S}=\operatorname{Supp} \mathcal{D}_{\pi_{1}} \cap E_{1}$ (in view of Proposition 4-(4)). Applying induction hypothesis to $Q_{1}$ we deduce that $F_{1}$ is dicritical for $\mathcal{L}_{1}^{\eta}$ and hence $F$ is dicritical for $\mathcal{L}^{\eta}$.

Let us prove the existence of a weak logarithmic model; in view of the above Theorem 14, this will give the stated Theorem 3 .

We need the following computation of Camacho-Sad indices at the non-singular curves of $\mathcal{S}$. We include a proof for the sake of completeness.

Lemma 15. Let $\mathcal{L}^{\eta}$ be the foliation of $\left(\mathbb{C}^{2}, 0\right)$ defined by $\eta=0$, with

$$
\eta=\frac{d y}{y}+\sum_{i=2}^{r} \lambda_{i} \frac{d f_{i}}{f_{i}}+\alpha, \quad \alpha \text { holomorphic, } d \alpha=0
$$


Then $I_{0}\left(\mathcal{L}^{\eta}, y=0\right)=-\sum_{i=2}^{r} \lambda_{i} \imath_{0}\left(f_{i}, y\right)$, where $I_{0}\left(\mathcal{L}^{\eta}, y=0\right)$ is the Camacho-Sad index and $\imath_{0}\left(f_{i}, y\right)$ denotes the intersection multiplicity.

Proof: (See also $[\mathbf{9}],[\mathbf{1 1}]$ for the non-dicritical case). We do induction on the number of blowing-ups needed to desingularize $\mathcal{L}^{\eta}$. If the origin is non-singular, then $I_{0}\left(\mathcal{L}^{\eta}, y=0\right)=0$ and $r=1$. If it is a simple singularity, then $r=2$ and $f_{2}$ is a coordinate $x=f_{2}$, in this case $-\lambda_{2}$ is the ratio of eigenvalues that gives the Camacho-Sad index. Now, let us do a blowing-up $\pi_{1}$ and consider the origin $P_{1}$ of the first chart, with coordinates $x^{\prime}=x, y^{\prime}=y / x$. Assume that the $f_{i}=0$ tangent to $y=0$ are those for $i \in B$ and denote $f_{i}^{\prime}=x^{\prime-m_{i}} f_{i} \circ \pi_{1}$ the strict transforms at $P_{1}$ of $f_{i}$. Now we have

$$
\pi_{1}^{*} \eta=\frac{d y^{\prime}}{y^{\prime}}+\left(1+\sum_{i=2}^{r} m_{i} \lambda_{i}\right) \frac{d x^{\prime}}{x^{\prime}}+\sum_{i \in B} \lambda_{i} \frac{d f_{i}^{\prime}}{f_{i}^{\prime}}+\alpha^{*},
$$

where $\alpha^{*}$ is closed and holomorphic at $P_{1}$. In view of the results of $[\mathbf{1}]$, we know that $I_{0}\left(\mathcal{L}^{\eta}, y=0\right)=I_{P_{1}}\left(\mathcal{L}_{1}^{\eta}, y^{\prime}=0\right)+1$ and by induction hypothesis

$$
I_{P_{1}}\left(\mathcal{L}_{1}^{\eta}, y^{\prime}=0\right)=-\left(1+\sum_{i=2}^{r} m_{i} \lambda_{i}+\sum_{i \in B} \lambda_{i} \imath_{P_{1}}\left(f_{i}^{\prime}, y^{\prime}\right)\right) .
$$

We end since $\imath_{0}\left(f_{i}, y\right)=m_{i}$ if $i \notin B$ and $\imath_{0}\left(f_{i}, y\right)=m_{i}+\imath_{P_{1}}\left(f_{i}^{\prime}, y^{\prime}\right)$ for $i \in B$.

Theorem 16. There is a weak logarithmic model $\mathcal{D}=(\mathcal{S}, \lambda)$ for $(\mathcal{F}, D)$ at $P$, relatively to $\mathcal{S}$.

Proof: Let us do induction on $N_{P}(\mathcal{F}, D)$. The case $N_{P}(\mathcal{F}, D)=0$ is done just by looking at the local situations. Let us consider the induction step.

Assume first that $\pi_{1}$ is non-dicritical for $\mathcal{F}$. Recall that $C_{P} \mathcal{F}=$ $E_{1} \cap \operatorname{Sing} \mathcal{F}_{1}=E_{1} \cap \operatorname{Sing}\left(\mathcal{F}_{1}, D_{1}\right)$ and by Proposition 4 we have $C_{P} \mathcal{F}=$ $C_{P} \mathcal{S}$. Put $C_{P} \mathcal{S}=\left\{P_{s}\right\}_{s=1}^{k}$ and $I_{s}=\left\{i ; P_{s} \in \Gamma_{1}^{i}\right\}$. The sets $\mathcal{S}^{(s)}=$ $\left\{E_{1}\right\} \cup\left\{\Gamma_{1}^{i}\right\}_{i \in I_{s}}$ are representative sets of separatrices for $\left(\mathcal{F}_{1}, D_{1}\right)$ at $P_{s}$. By induction hypothesis, we get a list of residues $\lambda^{(s)}=\{1\} \cup$ $\left\{\lambda_{i}\right\}_{i \in I_{s}}$ such that $\mathcal{D}^{(s)}=\left(\mathcal{S}^{(s)}, \lambda^{(s)}\right)$ is a weak logarithmic model for $\left(\mathcal{F}_{1}, D_{1}\right)$ at $P_{s}$. Let us define $\lambda=\left\{\lambda_{i}\right\}_{i=1}^{r}$. We are going to show that $\mathcal{D}=(\mathcal{S}, \lambda)$ is a weak logarithmic model for $(\mathcal{F}, D)$ at $P$. Let $\mathcal{L}^{(s)}$ be a logarithmic foliation defined by a $\mathcal{D}^{(s)}$-logarithmic form at $P_{s}$. Then, by Theorem $14, I_{P_{s}}\left(\mathcal{L}^{(s)}, E_{1}\right)=I_{P_{s}}\left(\mathcal{F}_{1}, E_{1}\right)$ and by Lemma 15 , we have 
that $I_{P_{s}}\left(\mathcal{L}^{(s)}, E_{1}\right)=-\sum_{i \in I_{s}} \lambda_{i} m_{i}$. Thus

$$
-1=\sum_{i=1}^{k} I_{P_{i}}\left(\mathcal{F}_{1}, E_{1}\right)=-\sum_{i=1}^{k} m_{i} \lambda_{i} .
$$

Hence $\lambda_{0}=\sum_{i=1}^{k} m_{i} \lambda_{i}=1$ and thus $\mathcal{D}_{\pi_{1}}=\mathcal{D}^{(s)}$ locally at $P_{s}$. Up to apply induction, we have to show the following properties:

(1) $\mathcal{D}$ is 1-faithful and $E_{1}$ is in the support of $\mathcal{D}_{\pi_{1}}$. This follows since $\lambda_{0} \neq 0$.

(2) $\operatorname{Sing}\left(\mathcal{D}_{\pi_{1}}, D_{1}\right)=\operatorname{Sing}\left(\mathcal{F}_{1}, D_{1}\right) \cap E_{1}$. This set is the tangent cone $C_{P} \mathcal{S}$.

Let us assume now that $\pi_{1}$ is $\mathcal{F}$-dicritical. Take a point $Q \in C_{P} \mathcal{S}$ and consider the set $\mathcal{S}_{Q}=\left\{\Gamma_{1}^{i}\right\}_{i \in I_{Q}}$ corresponding to the curves $\Gamma^{i}$ in $\mathcal{S}$ such that $Q \in \Gamma_{1}^{i}$. By induction hypothesis applied to $\left(\mathcal{F}_{1}, D_{1}\right)$ at $Q$, we get a $\mathbb{C}$-divisor $\mathcal{D}_{Q}^{*}=\left(\mathcal{S}_{Q}, \lambda_{Q}^{*}\right)$ that is a weak logarithmic model. Consider $\mu_{Q}=\sum_{i \in I_{Q}} m_{i} \lambda_{i}^{*}$ and let us prove that $\mu_{Q} \neq 0$. Take the 1 -form $\eta_{Q}$ at $P$ given by

$$
\eta_{Q}=\sum_{i \in I_{Q}} \lambda_{i}^{*} \frac{d f_{i}}{f_{i}}
$$

and assume that $\mu_{Q}=0$. Then $\pi_{1}^{*} \eta_{Q}$ defines a $\mathcal{D}_{Q}^{*}$-logarithmic foliation $\mathcal{L}_{Q}^{*}$ and, in view of Theorem 14 , we know that $\left(\mathcal{L}_{Q}^{*}, D_{1}\right)$ dominates $\left(\mathcal{F}_{1}, D_{1}\right)$ at $Q$. In particular, the exceptional divisor $E_{1}$ is dicritical for $\mathcal{L}_{Q}^{*}$. But this contradicts Proposition 10. Hence $\mu_{Q} \neq 0$. Now, since $C_{P} \mathcal{S}$ has at least two points (Proposition 4) we can take non-null coefficients $\epsilon_{Q}$ such that $\sum_{Q \in C_{P} \mathcal{S}} \epsilon_{Q} \mu_{Q}=0$. Put $\lambda_{i}=\epsilon_{Q} \lambda_{i}^{*}$ if $i \in I_{Q}$. Then the $\mathbb{C}$-divisor $\mathcal{D}=\left(\mathcal{S}, \lambda=\left\{\lambda_{i}\right\}_{i=1}^{r}\right)$ gives the desired weak logarithmic model.

\section{The escape set}

Assume that $\mathcal{D}=(\mathcal{S}, \lambda)$ is a weak logarithmic model for $(\mathcal{F}, D)$. Let $\eta$ be a $\mathcal{D}$-logarithmic 1 -form and $\mathcal{L}^{\eta}$ the foliation $\eta=0$. Two natural questions arise

(1) Once we have fixed the representative set of separatrices $\mathcal{S}$, determine the projective classes of $\lambda$ that give a weak logarithmic model for $(\mathcal{F}, D)$.

(2) Determine the "extra singularities" that can appear in the reduction of singularities of $\left(\mathcal{L}^{\eta}, D\right)$.

Some answers to these questions are given by the properties of the escape set. 
Let $\sigma: M^{*} \rightarrow(M, P)$ be a $\mathcal{D}$-admissible morphism. Next proposition is devoted to define and give the first properties of the escape set $\operatorname{Esc}_{\sigma} \mathcal{D}$. Let us first define $\operatorname{Esc}_{\sigma, \mathcal{D}}(\eta)$ for a $\mathcal{D}$-logarithmic 1-form $\eta$. We say that $Q \in E^{*}=\sigma^{-1}(P)$ is in the escape set $\operatorname{Esc}_{\sigma, \mathcal{D}}(\eta)$ if and only if $Q \notin$ $\operatorname{Supp} \mathcal{D}_{\sigma}$ and $\sigma^{*}(\eta)$ is either singular or does not have normal crossings with $E^{*}$ at $Q$. Note that if $Q \notin \operatorname{Supp} \mathcal{D}_{\sigma}$ then $\sigma^{*} \eta$ defines a closed holomorphic form, and hence $\sigma^{*} \eta=d h$, for a local holomorphic function $h$ at $Q$.

Proposition 17. Assume that $\mathcal{D}=(\mathcal{S}, \lambda)$ is faithful and let $\sigma: M^{*} \rightarrow$ $(M, P)$ be a $\mathcal{D}$-admissible sequence of blowing-ups. Consider two $\mathcal{D}$ logarithmic 1-forms $\eta$ and $\eta^{*}$. Then $\operatorname{Esc}_{\sigma, \mathcal{D}}(\eta)=\operatorname{Esc}_{\sigma, \mathcal{D}}\left(\eta^{*}\right)$. (We call this set $\left.\operatorname{Esc}_{\sigma} \mathcal{D}\right)$. Moreover, for any component of $F^{*}$ of $E^{*}=\sigma^{-1}(P)$ not contained in $\operatorname{Supp} \mathcal{D}_{\sigma}$ the set $F^{*} \cap \operatorname{Esc}_{\sigma} \mathcal{D}$ has at most $k-2$ points, where $k$ is the number of points in $F^{*} \cap \operatorname{Supp} \mathcal{D}_{\sigma}$.

Proof: Working by induction on the number of blowing-ups, it is enough to prove the following statement:

Assume that the first blowing-up $\pi_{1}$ produces a divisor $E_{1}$ not contained in $\operatorname{Supp} \mathcal{D}_{\pi_{1}}$. Then $E_{1} \cap \operatorname{Esc}_{\pi_{1}, \mathcal{D}}(\eta)$ depends only on $\mathcal{D}$ and has no more than $k_{P} \mathcal{S}-2$ points, where $k_{P} \mathcal{S}$ is the number of elements in the tangent cone $C_{P} \mathcal{S}$.

Let us prove this statement. Take the precedent notations and assume that

$$
\eta=\sum_{i=1}^{r} \lambda_{i} \frac{d f_{i}}{f_{i}}+\alpha,
$$

where $0=\lambda_{0}=\sum_{i=1}^{r} \lambda_{i} m_{i}$ and $\nu_{P}(f \eta)=m-1$. Up to a choice of suitable coordinates, we may assume that $\operatorname{In}^{m_{i}} f_{i}=\left(y+c^{i} x\right)^{m_{i}}$ with $c^{i}=c_{s}$ if $i \in I_{s}$, where the $I_{s}$ are pairwise disjoint and $\{1,2, \ldots, r\}=$ $\cup_{s=1}^{k} I_{s}$. Note that $k=k_{P} \mathcal{S}$. Denote $n_{s}$ the number of elements of $I_{s}$. Let us write $f \eta=A d x+B d y$. Note that $x \operatorname{In}^{m-1} A=-y \operatorname{In}^{m-1} B \neq 0$. Moreover $\operatorname{In}^{m-1} B=H(x, y) K(x, y)$, where

$$
\begin{aligned}
& H(x, y)=\prod_{i=1}^{r}\left(y+c^{i} x\right)^{m_{i}-1} \prod_{s=1}^{k}\left(y+c_{s} x\right)^{n_{s}-1} \\
& K(x, y)=\sum_{s=1}^{k} \mu_{s} \prod_{l=1, l \neq s}^{k}\left(y+c_{l} x\right)
\end{aligned}
$$

and $\mu_{s}=\sum_{i \in I_{s}} \lambda_{i} m_{i}$. Note that $K(1, y)$ is a polynomial of degree $\leq k-2$ (recall that $x$ divides $K(x, y)$ ). Taking coordinates $\left(x^{\prime}, y^{\prime}\right)$ in the 
first chart of the blowing-up, the points in the escape set are exactly the points not in the support such that $x^{\prime}=0$ and $K\left(1, y^{\prime}\right)=0$, hence, their position depend only on $\mathcal{D}$ and they are no more than $k-2$. Moreover, if $K(1, y)$ is of degree exactly $k-2$, then the origin of the second chart is not in the escape set.

Consider the transform $\mathcal{L}_{\sigma}^{\eta}$ of $\mathcal{L}^{\eta}$ by $\sigma$. Note that $\mathcal{L}_{\sigma}^{\eta}$ is given by $\sigma^{*} \eta=$ 0 . Let $F^{*}$ be a component of $E^{*}=\sigma^{-1}(P)$ not contained in Supp $\mathcal{D}_{\sigma}$ (recall that in view of Proposition 13 this is equivalent to say that $F^{*}$ is a dicritical component for $\mathcal{L}_{\sigma}^{\eta}$ ). Consider a point $Q \in F^{*} \backslash \operatorname{Supp} \mathcal{D}_{\sigma}$. If $Q \notin \operatorname{Esc}_{\sigma} \mathcal{D}$ then $\sigma^{*} \eta$ is non-singular and transversal to $F^{*}$ and hence $Q \notin \operatorname{Sing}\left(\mathcal{L}_{\sigma}^{\eta}, \sigma^{-1}(P)\right)$. Thus, if we define

$$
\operatorname{Esc}_{\sigma}\left(\mathcal{L}^{\eta}\right)=\operatorname{Sing}\left(\mathcal{L}_{\sigma}^{\eta}, \sigma^{-1}(P)\right) \backslash \operatorname{Supp} \mathcal{D}_{\sigma},
$$

we get that $\operatorname{Esc}_{\sigma}\left(\mathcal{L}^{\eta}\right) \subset \operatorname{Esc}_{\sigma}(\mathcal{D})$. But the converse is not true, as we will show in some examples.

In the case of a weak logarithmic model, taking $\sigma=\pi$, we see that

$$
\operatorname{Esc}_{\pi}\left(\mathcal{L}^{\eta}\right)=\operatorname{Sing}\left(\mathcal{L}^{\prime}, D^{\prime}\right) \backslash \operatorname{Sing}\left(\mathcal{F}^{\prime}, D^{\prime}\right) .
$$

Corollary 18. For any $\mathcal{F}^{\prime}$-dicritical component $F^{\prime}$ of $D^{\prime}$, the number of points in $\operatorname{Esc}_{\pi}\left(\mathcal{L}^{\eta}\right) \cap F^{\prime}$ is smaller or equal than $v_{D^{\prime}} F^{\prime}-2$.

Proof: It is enough to note that for any $\mathcal{F}^{\prime}$-dicritical component $F^{\prime}$ of $D^{\prime}$, the points of $F^{\prime}$ in the support $\operatorname{Supp} \mathcal{D}_{\pi}$ coincide with the intersections of $F^{\prime}$ with the other components of $D^{\prime}$.

In order to get a weak logarithmic model, the escape set $\mathrm{Esc}_{\pi} \mathcal{D}$ can be fixed to be an arbitrarily chosen point in each $\mathcal{F}^{\prime}$-dicritical component $F^{\prime}$ of $E^{\prime}$ of valence $v_{D^{\prime}} F^{\prime} \geq 3$. Moreover, this produces a unique choice of the projective class of $\lambda$. Let us outline the proof of this statement. Take the notations as in the proof of Proposition 17. Recall the polynomial

$$
K(x, y)=\sum_{s=1}^{k} \mu_{s} \prod_{l=1, l \neq s}^{k}\left(y+c_{l} x\right)
$$

where $\mu_{s}=\sum_{i \in I_{s}} \lambda_{i} m_{i}$. There is a unique choice of the $\mu_{s}$ such that $K(1, y)$ is a non-null constant polynomial. This gives by induction the desired selection of $\lambda$, by putting the escape point at the infinity of the first chart of the blowing-up. More generally, we can fix $v_{D^{\prime}} F^{\prime}-2$ points with multiplicity in each $F^{\prime}$ to get a unique projective class of $\lambda$.

Example 19 (One dicritical blowing-up). The case that we get a nonsingular foliation under the first (dicritical) blowing-up has been considered by M. Klughertz [7], [8]. She provides a rational model for the 
equisingularity type of the foliation, but her presentation is not in terms of a representative set of separatrices as we propose. In our terms, this case corresponds for instance to foliations having separatrices $f_{i}=0$ for

$$
f_{i}=\left(y+c_{i} x\right)^{m_{i}}+x^{m_{i}+1}, \quad i=1,2, \ldots, r .
$$

Let us select $\lambda=\left\{\lambda_{i}\right\}_{i=1}^{r}$ such that the escape set of the $\mathbb{C}$-divisor is in the infinity. If all the $m_{i}=n$, one can verify by a long but elementary computation that $x^{r-2}$ divides $f \eta$ and we get as consequence that $\operatorname{Esc}_{\pi} \mathcal{L}=\emptyset$. Nevertheless, already in the case $m_{i}=2$ and $r=5$, if we consider the separatrices given by $\left(y+c_{i} x\right)^{2}+\delta_{i} x^{3}=0$ for general $\delta_{i}$, we get $\operatorname{Esc}_{\pi} \mathcal{L} \neq \emptyset$.

Acknowledgements. We are indebted to J. M. Aroca, J.-F. Mattei and D. Marín for fruitful conversations on the subject. We also thank the referee for his contribution to a good presentation of the paper.

\section{References}

[1] C. Camacho and P. Sad, Invariant varieties through singularities of holomorphic vector fields, Ann. of Math. (2) 115(3) (1982), 579-595.

[2] C. Camacho, A. Lins Neto and P. Sad, Topological invariants and equidesingularization for holomorphic vector fields, J. Differential Geom. 20(1) (1984), 143-174.

[3] F. CANO, Reduction of the singularities of codimension one singular foliations in dimension three, Ann. of Math. (2) 160(3) (2004), 907-1011.

[4] D. Cerveau and J.-F. Mattei, Formes intégrables holomorphes singulières, Astérisque 97 (1982), 193 pp.

[5] N. Corral, Sur la topologie des courbes polaires de certains feuilletages singuliers, Ann. Inst. Fourier (Grenoble) 53(3) (2003), $787-814$.

[6] N. Corral and P. Fernández-Sánchez, Isolated invariant curves of a foliation, Proc. Amer. Math. Soc. (to appear).

[7] M. Klughertz, Feuilletages holomorphes à singularité isolée ayant une infinité de courbes intégrales, Thèse, Univ. Paul Sabatier, Toulouse (1988).

[8] M. Klughertz, Existence d'une intégrale première méromorphe pour des germes de feuilletages à feuilles fermées dans le plan complexe, Topology 31(2) (1992), 255-269.

[9] A. Lins Neto, Algebraic solutions of polynomial differential equations and foliations in dimension two, in: "Holomorphic dynamics" 
(Mexico, 1986), Lecture Notes in Math. 1345, Springer, Berlin, 1988, pp. 192-232.

[10] J.-F. Mattei And R. Moussu, Holonomie et intégrales premières, Ann. Sci. École Norm. Sup. (4) 13(4) (1980), 469-523.

[11] P. Rouillé, Courbes polaires et courbure, Thèse, Univ. Bourgogne, Dijon (1996).

[12] A. SeIDEnBERG, Reduction of singularities of the differential equation $A d y=B d x$, Amer. J. Math. 90 (1968), 248-269.

\section{Felipe Cano:}

Departamento de Álgebra, Geometría y Topología

Universidad de Valladolid

Facultad de Ciencias

47005-Valladolid

Spain

E-mail address: fcano@agt.uva.es

Nuria Corral:

Departamento de Matemática Aplicada I

Universidad de Vigo

Escuela U. de Ingeniería Técnica Forestal. Campus A

Xunqueira. 36005 Pontevedra

Spain

E-mail address: ncorral@uvigo.es

Primera versió rebuda el 7 de febrer de 2005, darrera versió rebuda el 17 d'octubre de 2005. 